\title{
AVALIAÇÃO TOMOGRÁFICA E TENSIOMÉTRICA DE FÊMURES DE RATAS OOFORECTOMIZADAS SUBMETIDAS À TERAPIA DE REPOSIÇÃO HORMONAL
}

\author{
TOMOGRAFIC AND TENSIOMETRIC ASSESSMENT OF FEMURS OF \\ OOPHORECTOMIZED RATS SUBMITTED TO HORMONE REPLACEMENT THERAPY
}

Fábio Alexandre Martynetz', Maria de Lourdes Pessole Biondo-Simões², Juliano Rodrigo Martynetz ${ }^{3}$, Tatiana Daher Martynetz ${ }^{4}$, Elise Zimerman ${ }^{5}$, Heraldo Mello Neto ${ }^{6}$

\section{RESUMO}

Objetivo: Analisar os efeitos da TRH na avaliação biomecânica e tomográfica dos fêmures de ratas submetidas à menopausa induzida. Métodos: Foram divididas igualmente em três grupos 45 ratas Wistar adultas. O primeiro e o segundo constituíram-se de ratas ooforectomizadas e o terceiro, o grupo controle, de ratas não ooforectomizadas. Após a constatação da falência hormonal (citologia esfoliativa). somente o primeiro grupo recebeu TRH, durante dois meses. Após esse período, os fêmures foram desarticulados e submetidos a testes biomecânicos através de máquina universal de ensaios para avaliação da resistência e submetidos a tomografia para determinação da densidade óssea. Resultados: A citologia esfoliativa demonstrou indução de falência hormonal em todos os animais ooforectomizados. Notou-se diferença significante $(p=0,030)$ entre os grupos ao se mensurar a força máxima de resistência, maior no grupo que recebeu TRH. Observou-se maior fragilidade óssea nos animais ooforectomizados sem TRH quando comparados com os com TRH $(p=0,010)$ em relação ao grupo controle $(p=0,0107)$. Houve aumento da resistência óssea nas ratas ooforectomizadas com TRH em relação às sem TRH e valores semelhantes aos do grupo controle $(\mathrm{p}=$ 0,179 ). Na avaliação tomográfica, não se encontraram diferenças significantes entre os grupos $(\mathrm{p}=0,625)$. Conclusão: Observouse aumento significativo da resistência óssea com a utilização de TRH. No entanto, o tratamento com TRH não apresentou alteração significante da densidade mineral óssea.

Descritores - Menopausa; Terapia de reposição hormonal; Tomografia; Osso e ossos; Osteoporose; Fêmur; Ratos

\section{ABSTRACT}

Objective: To analyze the effects of Hormone Replacement Therapy on the biomechanical and tomographic evaluation of femurs of rats submitted to induced menopause. Methods: Forty-five adult Wistar rats were divided equally into three groups. The first and second groups consisted of rats submitted to oophorectomy, and the third was the control group, consisting of non-oophorectomized rates. After observing hormonal failure (exfoliative cytology), only the first group received HRT, over a two-month period. After this period, the femurs were disarticulated and submitted to biomechanical tests in a universal testing machine to evaluate stamina, and submitted to tomographic evaluation to determine mineral bone density. Results: The exfoliative cytology showed induction of hormonal failure in all the animals oophorectomized. A significant difference ( $p=0.030)$ was observed between the groups in the measurements of maximum stamina, which was higher in the group with HRT. Greater bone fragility was observed in the oophorectomized animals that did not receive HRT, compared with those that received HRT $(p=0.010)$ in relation to the control group ( $p=0.0107)$. There was in increase in bone strength in the oophorectomized rats with HRT compared with those without HRT, and similar values to the control group ( $p=1.179$ ). In the tomographical evaluation, no significant differences were found between the groups ( $p=0.625$ ). Conclusion: A significant increase in bone strength was observed with the use of HRT. However, treatment with HRT did not show any significant alteration in bone mineral density.

Keywords - Menopause; Hormone replacement therapy; Tomography; Bone and bones; Osteoporosis; Femur; Rats

\footnotetext{
1 - Mestrando do Programa de Pós-Graduação em Clínica Cirúrgica da PUCPR.

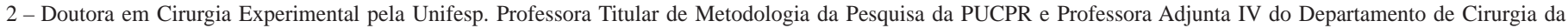
Universidade Federal do Paraná.

3 - Residente em Ortopedia e Traumatologia no Serviço do Hospital Universitário Cajuru (PUCPR).

4 - Médica Oftalmologista, graduada pela Pontifícia Universidade Católica do Paraná.

5 - Médica Otorrinolaringologista, graduada pela Pontifícia Universidade Católica do Paraná.

6 - Médico Neurorradiologista da X-leme. Radiologista do Serviço de Imagem do Hospital Universitário Cajuru e da Santa Casa de Misericórdia de Curitiba (PUCPR)
} 


\section{INTRODUÇÃO}

A osteoporose é uma doença óssea sistêmica, associada à alteração da arquitetura óssea e à redução da massa óssea, que gera aumento do número de fraturas, principalmente em mulheres pós-menopausadas ${ }^{(1)}$.

Em torno de 50\% dessas mulheres poderão desenvolver algum tipo de fratura relacionada com a alteração da qualidade óssea e 15\% poderão ter fratura de fêmur ${ }^{(2)}$. Esta apresenta alta prevalência em mulheres acima de 49 anos $(32,7 \%)^{(3)}$.

As fraturas, principalmente as do fêmur, em pacientes com osteoporose fazem parte do cotidiano do ortopedista, tendo em vista o aumento de sua incidência concomitante à elevação da expectativa de vida.

São fraturas provocadas ou agravadas pela baixa qualidade óssea, limitando assim os métodos de tratamento. Apenas 30\% dos pacientes vítimas de fratura por osteoporose conseguem retomar a mesma qualidade de vida sem alguma limitação funcional, e a mortalidade nos primeiros seis meses chega a $23,3 \%^{(4)}$.

A prevenção da perda óssea iniciada logo após a menopausa serviria para reduzir a incidência de fraturas numa fase mais tardia da vida da mulher ${ }^{(5)}$.

A terapia de reposição hormonal (TRH), seja oral, intradérmica ou parenteral, tem sido demonstrada útil na prevenção da perda óssea e na redução do risco de fraturas no climatério ${ }^{(6,7)}$.

Mulheres que iniciaram o uso de estrógeno antes dos 60 anos e continuaram com a TRH mantiveram a densidade mineral óssea, perderam menos osso e tiveram $37 \%$ menos risco de fratura $^{(8)}$.

A tomografia computadorizada é uma grande ferramenta na prática médica. Uma de suas propriedades é a capacidade de qualificar e quantificar a arquitetura óssea, permitindo melhor avaliação morfológica ${ }^{(9)}$.

A terapia de reposição hormonal pode aumentar a massa óssea, diminuir sua perda e o risco de fraturas, assim como suas sequelas ${ }^{(3,4,10,11)}$.

\section{MÉTODOS}

Foram utilizadas 45 ratas (Rattus norvegicus albinus, Rodentia mammalia) da linhagem Wistar-PUCPR, com idade entre 90 e 100 dias, pesando entre 150 e 200 gramas que foram fornecidas pelo Biotério Central da Pontifícia Universidade Católica do Paraná. As ratas foram mantidas em condições ambientais constantes, com ciclo dia/noite de 12 horas e temperatura ambiente con- trolada de $20 \pm 2^{\circ} \mathrm{C}$. A umidade relativa do ar e o ruído eram os próprios do ambiente. Tiveram livre acesso à água e à ração própria para a espécie. Foram alojadas cinco ratas em cada gaiola e todas ficaram dispostas à igual distância da fonte de luz.

A amostra foi dividida em três grupos, com 15 animais cada um. O primeiro e o segundo grupos constituíram-se de ratas ooforectomizadas e o terceiro, o grupo controle, de ratas hígidas não submetidas a tal procedimento. As pertencentes ao grupo 1 receberam reposição hormonal.

Inicialmente, os animais, sob anestesia, foram submetidos à laparotomia mediana, sendo realizada ooforectomia bilateral nas ratas dos grupos 1 e 2 . As do grupo 3 sofreram uma laparotomia simuladora.

Foi coletado material para esfregaço vaginal, para a determinação do padrão hormonal de cada um dos animais que constituíram a amostra, no início do estudo, por meio da técnica de Papanicolau.

Após 28 dias do procedimento cirúrgico, foi colhido um segundo esfregaço vaginal dos animais dos três grupos, com a finalidade de confirmar a carência hormonal.

Detectada a "menopausa” induzida, iniciou-se a terapia de reposição hormonal diariamente, da seguinte forma: os animais que compunham o grupo 1 receberam 50 $\mu \mathrm{g}$ de estrogênio conjugado associado a $2 \mathrm{mg}$ de acetato de medroxiprogesterona ao dia. A medicação foi feita por meio de sonda orogástrica. As ratas dos grupos 2 e 3 receberam igual volume de solução de $\mathrm{NaCl}$ a $0,9 \%$ pela mesma via.

No $15^{\circ}$ dia, foi realizada nova coleta de esfregaços vaginais dos animais dos três grupos, para confirmação do estado hormonal.

Após dois meses de terapia procedeu-se à eutanásia com dose letal de tiopental sódico, intraperitoneal. Constatado o óbito, foi feita a retirada do fêmur direito, que foi submetido a estudo tomográfico e de resistência.

$\mathrm{O}$ estudo de imagem foi feito com um aparelho de tomografia da marca Siemens ${ }^{\circledR}$ Somatom, modelo Esprit, produzido em Erlanger, Alemanha. Foram realizados cortes tomográficos do fêmur, que permitiram a avaliação de três regiões com dois cortes de 1,5mm de espessura em cada área: 1) região proximal que incluía desde a região subcapital do colo até o trocanter menor; 2) região diafisária do istmo até um centímetro distal; e 3) metáfise distal (Figura 1). 


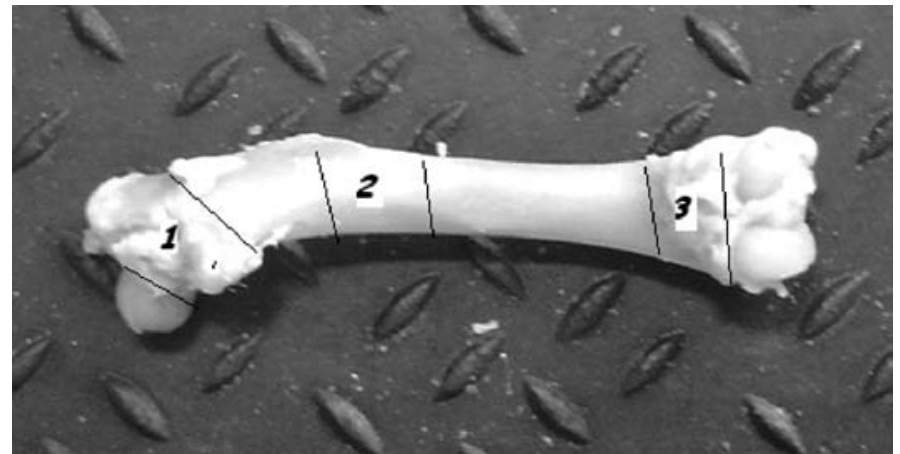

Figura 1 - Demonstração das áreas do fêmur que foram analisadas pela tomografia computadorizada

Em cada área foi analisada a densidade óssea por meio da relação da estrutura óssea cortical e medular (Figura 2).

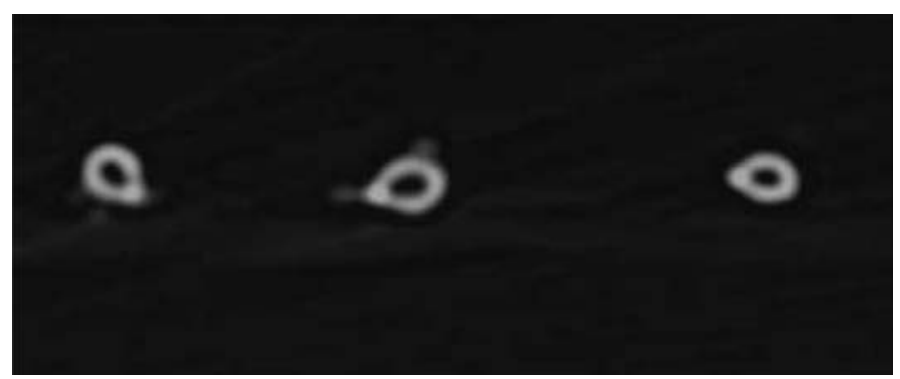

Figura 2 - Aspectos dos cortes tomográficos em axial, no segmento médio, nos três grupos

Foi utilizada a unidade de Hounsfield (UH) para a mensuração da densidade óssea, unidade de medida de densidade utilizada em tomografia computadorizada, com escala empírica que identifica a penetrabilidade da radiação diagnóstica de acordo com o tipo de tecido.

A resistência foi avaliada em um ensaio destrutivo realizado no Laboratório de Ensaios Destrutivos do Departamento de Engenharia Mecânica, no Parque Tecnológico da Pontifícia Universidade Católica do Paraná.
Os fêmures foram submetidos a um ensaio destrutivo em um aparelho de ensaio de força de flexão em três pontos (tensão/ compressão), da marca Emic ${ }^{\circledR}$ de fabricação nacional modelo $D L-500$ (Figura 3). Tal dispositivo era conectado a um microcomputador com software M-test de ensaio mecânico, por meio de um canal serial padrão Rs-232. Esse software possibilita a conversão dos valores numéricos absolutos em unidade de newtons, assim avaliando a força necessária para fraturar um determinado material.

Para realizar a medida no aparelho de ensaio mecânico, foi necessária a confecção de uma estrutura em aço para apoio das diáfises dos fêmures. Cada apoio de um centímetro foi posicionado na região metafisária dos fêmures e a força de flexão foi realizada por uma terceira força contrária, também adaptada por uma ponteira de aço de $0,5 \mathrm{~cm}$. A medida dos apoios laterais foi empírica, visto que não influencia no teste; a ponteira de $0,5 \mathrm{~cm}$ foi determinada pela média da medida do diâmetro da secção transversa do eixo dos fêmures examinados ${ }^{(12)}$. A força era aplicada até se obter a fratura (Figura 3).

Os resultados obtidos foram submetidos à análise estatística. Para a comparação dos grupos em relação às variáveis do estudo que atenderam à condição de normalidade, foi usada a análise de variância com um fator.

As comparações múltiplas foram feitas usando-se o teste LSD. Para variáveis que apresentaram assimetria na sua distribuição, as comparações entre os grupos foram feitas utilizando-se o teste não paramétrico de Kruskall-Wallis.

Para avaliar a condição de normalidade das variáveis foi usado o teste de Shapiro-Wilks e para avaliar a homogeneidade das variâncias, o teste de Levène.

A associação entre variáveis quantitativas foi avaliada estimando-se o coeficiente de correlação de Spearman. Valores de $\mathrm{p}<0,05$ indicaram significância estatística.
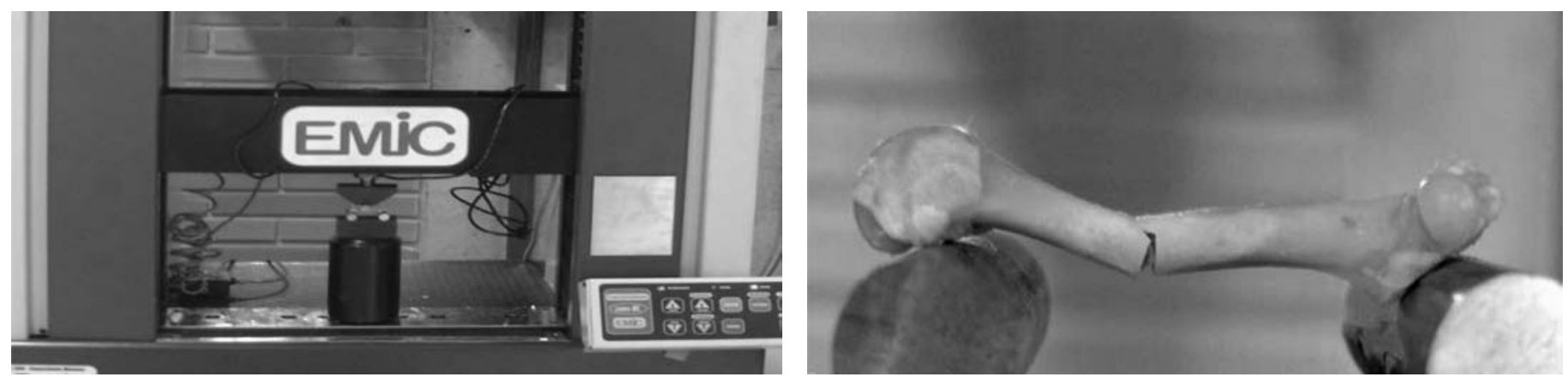

Figura 3 - Demonstração do posicionamento da peça no aparelho para a produção da fratura à esquerda e padrão de fratura obtido à direita 


\section{RESULTADOS}

No 15. ${ }^{\circ}$ dia após a indução de “menopausa” verificouse, nos esfregaços vaginais, que todas as ratas ooforectomizadas estavam em fase de diestro, caracterizando presença de estado hormonal compatível com hipoestrogenismo.

Durante o tempo de estudo houve a morte de 16 animais. O grupo 1 apresentou o maior número de óbitos, restando apenas seis animais; no grupo 2 restaram 12 animais e 11 animais no grupo 3.

As 29 amostras restantes foram submetidas à análise tomográfica e ao teste de resistência de acordo com seus respectivos grupos.

Notou-se diferença significante $(p=0,030)$ entre os grupos ao se mensurar a força máxima no ensaio de resistência, mais acentuada no grupo que recebeu terapia de reposição hormonal em relação àquele que não foi tratado (Quadro 1). Observou-se que o grupo 2 apresentou maior fragilidade no ensaio de resistência em relação ao grupo $1(\mathrm{p}=0,010)$ e ao grupo $3(\mathrm{p}=0,0107)$.

Quadro 1 - Valores em newtons para força máxima em cada grupo

\begin{tabular}{|c|c|c|c|c|c|c|}
\hline GRUPO & N & MÉDIA & MEDIANA & MíNIMO & MÁXIMO & DESVIO PADRÃO \\
\hline 1 & 6 & 47,22 & 48,61 & 36,48 & 57,61 & 7,49 \\
\hline 2 & 12 & 40,72 & 40,83 & 32,93 & 48,63 & 4,24 \\
\hline 3 & 11 & 43,98 & 43,86 & 39,52 & 49,80 & 3,03 \\
\hline
\end{tabular}

Teste LSD $p=0,030$

Ao analisar a força de ruptura sobre as peças do experimento observou-se diferença significante entre os grupos ( $\mathrm{p}=0,049)$ (Quadro 2), evidenciada mais claramente ao relacionar o grupo 1 com o grupo 2 (p $=0,016$ ). A força de ruptura era semelhante entre os animais que tiveram reposição hormonal e os controle ( $\mathrm{p}=0,179$ ). Os fêmures dos animais do grupo controle apresentaram maior resistência que os do grupo 2, sem significância estatística ( $\mathrm{p}=0,171)$.

Quadro 2 - Valores em newtons da força de ruptura nos três grupos

\begin{tabular}{|c|c|c|c|c|c|c|}
\hline GRUPO & $\mathbf{N}$ & MÉDIA & MEDIANA & MÍNIMO & MÁXIMO & DESVIO PADRÃO \\
\hline 1 & 6 & 46,30 & 47,70 & 35,55 & 57,15 & 7,72 \\
\hline 2 & 12 & 40,02 & 39,88 & 31,18 & 47,38 & 4,19 \\
\hline 3 & 11 & 42,88 & 43,16 & 36,91 & 49,72 & 3,55 \\
\hline
\end{tabular}

Teste LSD $p=0,049$

Em relação à avaliação da densidade óssea mineral média do fêmur, por meio da tomografia, observouse, nitidamente, uma tendência de semelhança em seus valores, não sendo, no entanto, estatisticamente significante $(\mathrm{p}=0,625)$ (Quadro 3).
Quadro 3 - Valores da densidade óssea mineral média (unidade Hounsfield)

\begin{tabular}{|c|c|c|c|c|c|c|}
\hline GRUPO & N & MÉDIA & MEDIANA & MÍNIMO & MÁXIMO & DESVIO PADRÃO \\
\hline 1 & 6 & 1,53 & 1,53 & 1,37 & 1,66 & 0,10 \\
\hline 2 & 12 & 1,63 & 1,65 & 1,26 & 1,97 & 0,21 \\
\hline 3 & 11 & 1,66 & 1,58 & 1,29 & 2,39 & 0,28 \\
\hline
\end{tabular}

Teste LSD $p=0,625$

Aqueles animais que receberam reposição hormonal apresentaram, na tomografia, menor densidade mineral no segmento proximal em relação aos outros dois grupos, não sendo estatisticamente significante $(p=0,073)$.

Os animais do grupo controle apresentaram maior densidade mineral, na tomografia, em relação aos dos grupos experimentos, no segmento médio e distal ( $\mathrm{p}=0,0437)$ (Quadro 4).

Quadro 4 - Valores da densidade óssea mineral no segmento médio do fêmur (unidade de Hounsfield

\begin{tabular}{|c|c|c|c|c|c|c|}
\hline GRUPO & N & MÉDIA & MEDIANA & MÍNIMO & MÁXIMO & DESVIO PADRÃO \\
\hline 1 & 6 & 1,51 & 1,46 & 1,39 & 1,68 & 0,12 \\
\hline 2 & 12 & 1,62 & 1,62 & 1,21 & 2,20 & 0,33 \\
\hline 3 & 11 & 1,75 & 1,66 & 1,17 & 2,39 & 0,41 \\
\hline
\end{tabular}

Teste LSD $p=0,0437$

No grupo 1, a força de ruptura não demonstrou correlação estatisticamente significante quando avaliada comparativamente com a densidade óssea mineral tomográfica. O mesmo fato pode ser notado naqueles fêmures dos animais que não foram submetidos à reposição hormonal, assim como no grupo controle (Quadro 5).

Quadro 5 - Correlação entre a densidade óssea mineral e a força de ruptura por meio do coeficiente de correlação de Spearman

\begin{tabular}{|c|c|c|c|}
\hline GRUPO & $\mathbf{N}$ & $\begin{array}{c}\text { COEF. DE CORRELAÇÃO DE } \\
\text { SPEARMAN }\end{array}$ & VALOR DE $\mathbf{p}$ \\
\hline 1 & 6 & $-0,257$ & 0,623 \\
\hline 2 & 12 & 0,137 & 0,672 \\
\hline 3 & 11 & $-0,118$ & 0,729 \\
\hline
\end{tabular}

A força máxima não apresentou correlação estatística significante ao compararmos com a densidade óssea tomográfica, nos elementos dos grupos 1 e 2, assim como nos animais do grupo controle (Quadro 6).

\section{DISCUSSÃO}

O homem é um ser bípede e seus fêmures estão dispostos de maneira a suportar cargas mais altas se comparado com o rato. Estruturalmente em sua disposição 
Quadro 6 - Demonstração da correlação entre densidade óssea mineral e a força máxima por meio do coeficiente de correlação de Spearman

\begin{tabular}{|c|c|c|c|}
\hline \multicolumn{4}{|c|}{ FORÇA MÁXIMA } \\
\hline Grupo & $\mathbf{N}$ & $\begin{array}{c}\text { Coef. de correlação de } \\
\text { Spearman }\end{array}$ & Valor de $\mathbf{p}$ \\
\hline 1 & 6 & $-0,086$ & 0,872 \\
\hline 2 & 12 & 0,294 & 0,353 \\
\hline 3 & 11 & $-0,145$ & 0,670 \\
\hline
\end{tabular}

morfológica e espacial observa-se uma divergência entre o eixo mecânico e anatômico dos membros inferiores, que oferece maior resistência as forças externas.

Ao envelhecer, o trabeculado ósseo diminui e observa-se uma área de fraqueza na região proximal do fêmur que, associada à disposição das linhas de força, traçãocompressão, na região do colo e pertrocanterianas, a torna mais suscetível a fraturas. Essas, por sua vez, foram descritas e classificadas de maneira radiológica, de acordo com o desaparecimento das linhas trabeculares ${ }^{(13)}$.

Albright, em 1941 apud Nordin e Polley ${ }^{(14)}$, correlacionou o desenvolvimento da osteoporose em mulheres na menopausa ao hipoestrogenismo. Segundo ele, esse estado de diminuição nos níveis séricos de estrógenos reduzia a formação óssea. Atualmente, sabe-se que a falta desse hormônio aumenta o turnover ósseo, aumentando tanto a formação quanto a reabsorção óssea.

Nos últimos dez anos a expectativa média de vida do brasileiro cresceu de 68,1 anos para 72,3 anos, principalmente das mulheres, que está estimada entre 72 e $76,1 \operatorname{anos}^{(15)}$. O aumento da população geriátrica leva à maior incidência de osteoporose ${ }^{(2)}$ e, consequentemente, ao aumento do risco de fratura nessa população.

A terapia de reposição hormonal pode aumentar a massa óssea, pela diminuição da perda por inibição da reabsorção óssea e tornar, teoricamente, o osso mais resistente. O estrógeno atua nesse sistema inibindo a reabsorção óssea, por induzir a apoptose dos osteoclastos, por meio de receptores alfa nas células ósseas, influenciando a formação, a manutenção e a reabsorção do tecido ósseo. Previne, assim, a perda óssea e pode diminuir o risco de fraturas ${ }^{(16)}$.

A ooforectomia, no presente estudo, promoveu a carência hormonal e levou à redução da DMO no segmento médio, além de diminuir a resistência óssea no ensaio destrutivo. Esse modelo mostrou-se eficiente para promover o estado de hipoestrogenismo. Cardoso Netto et al $^{(17)}$ já haviam demonstrado que a ooforectomia induz a menopausa e reduz o limite de deformação óssea, tornando o fêmur menos resistente.
Neste estudo, notou-se superioridade significativa da força máxima e de ruptura nos fêmures dos animais tratados com reposição hormonal em relação àqueles que não foram submetidos a tal tratamento. Assim, comprovou-se a ação protetora óssea da terapia de reposição hormonal.

O método de ensaio destrutivo com a aplicação de força em três pontos permitiu, de maneira segura, avaliar a resistência óssea. Esse método já havia sido utilizado anteriormente por Probst et al $^{(18)}$.

Atualmente, o diagnóstico da osteoporose é confirmado pela densitometria óssea. A densidade óssea reflete a quantidade de mineral quantificada numa área do esqueleto, representando a densidade mineral óssea, expressa em gramas pela área ou volume medidos. A densitometria de dupla energia baseada em raios X (DEXA) é uma técnica eficaz, sendo considerada hoje como o padrãoouro para a determinação da densidade óssea ${ }^{(19)}$.

Segundo Marshall et al ${ }^{(20)}$, a densitometria óssea mineral é um método que pode predizer o risco de fratura; no entanto não é capaz de identificar o indivíduo que a terá, não sendo recomendado o programa sistemático de rastreamento de osteoporose para mulheres menopausadas ${ }^{(20)}$.

A literatura consagrou a tomografia como um método de avaliação da densidade óssea, embora a densitometria seja hoje o exame padrão-ouro para a determinação da densidade óssea em humanos. Optou-se pelo estudo tomográfico visto que sua acurácia se assemelha à da DEXA e sua utilização em pequenos animais é bem definida na literatura ${ }^{(21)}$.

A análise óssea pela tomografia computadorizada é primordial na comprovação dos efeitos de reabsorção e formação óssea ${ }^{(22-24)}$, além de caracterizar fielmente a arquitetura óssea, propiciando melhor compreensão da morfologia em ratos ${ }^{(25)}$.

A tomografia tem a capacidade de estabelecer uma correlação entre as propriedades biomecânicas e a morfologia do osso, em fêmures de $\operatorname{ratos}^{(26,27)}$. Esse exame pode, ainda, refletir claramente os parâmetros das propriedades morfológicas do osso e ser utilizado em experimentos in vivo(28).

Lima et $a l^{(9)}$ demonstraram a eficácia da tomografia computadorizada em três dimensões na avaliação morfológica da estrutura óssea de fêmures de ratos Wistar; no entanto, o custo elevado desse aparelho e a nossa limitação de acesso a tal tecnologia permitiu apenas o uso de tomografia axial computadorizada convencional.

No presente estudo observou-se que a terapia de reposição, em ratas, levou a aumento significante da resistência óssea. No entanto, não se constatou alteração significativa do aumento da densidade óssea mineral nos fêmures dos animais submetidos à reposição hormonal. 
É possível que o método utilizado para essa avaliação não tenha apresentado sensibilidade suficiente para detectar possíveis alterações nessa amostragem.

Neste estudo, optou-se por realizar o ensaio de resistência óssea em terço médio diafisário do fêmur devido à impossibilidade de reproduzir o mecanismo de trauma e de fratura do segmento proximal, como acontece no homem. Optou-se pela avaliação do segmento médio, uma vez que essa é a área que foi submetida à ação direta da força durante o ensaio de três pontos.

O rato é um animal quadrúpede e a disposição de suas linhas de força, o seu eixo mecânico e anatômico não são os mesmos dos humanos, o que inviabilizou um ensaio torcional que reproduzisse uma fratura no segmento proximal, semelhante ao mecanismo de trauma que frequentemente atinge a população geriátrica.

O propósito do teste de flexão de três pontos foi estabelecer a relativa força do osso quando forças eram aplicadas como a flexão deformante sobre um eixo. O osso quebra-se primeiro em tensão e a fratura propaga-se para o lado da compressão, abrindo uma asa de borboleta ${ }^{(12,26)}$.

O modelo de ensaio destrutivo em três pontos é confiável e capaz de mensurar, em newtons, valores numé-

\section{REFERÊNCIAS}

1. Drugs \& Therapy Perspectives. Postmenopausal osteoporosis: optimum time to start therapy unclear. Drug Perspect. 1997;10(7):8-12.

2. Nelson HD, Rizzo J, Harris E, Cauley J, Ensrud K, Bauer DC, et al. Osteoporosis and fractures in women using estrogen. Arch Intern Med. 2002;162(20):2278-84.

3. Faisal-Cury A, Zacchello KP. Osteoporose: prevalência e fatores de risco em mulheres de clínica privada maiores de 49 anos de idade. Acta Ortop Bras. 2007;15(3):146-50.

4. Brandão CM, Lima MG, Silva AL, Silva GD, Guerra AA Jr, Acúrcio FA. Treatment of postmenopausal osteoporosis in women: a systematic review. Cad Saude Publica. 2008;24(Suppl 4):s592-606.

5. Cooper C, Fogelman I, Melton LJ 3rd. Bisphosphonates and vertebral fracture: an epidemological perspective. Osteoporosis Int. 1991;2(1):1-4.

6. Compston JE. Prevention and management of osteoporosis. Current trends and future prospects. Drugs. 1997;53(5):727-35.

7. Russo LAT. Osteoporose pós-menopausa: opções terapêuticas. Arq Bras Endocrinol Metab. 2001;45(4):401-6.

8. Cauley JA, Zmuda JM, Ensrud KE. Timing of estrogen replacement therapy for optimal osteoporosis prevention. J Clin Endocrinol Metab. 2001;86(12):5700-5.

9. Lima ICB, Oliveira LF, Lopes RT. Bone architecture analyses of rat femur with $3 \mathrm{D}$ microtomographics images. J Radioanal Nucl Chem. 2006;269(3):639-42.

10. Felson DT, Zhang Y, Hannan MT, Kiel DP, Wilson PW, Anderson JJ. The effect of postmenopausal estrogen therapy on bone density in elderly women. $\mathrm{N}$ Engl J Med. 1993;329(16):1141-6.

11. Araújo DV, Oliveira JHA, Bracco OL. Custo da fratura osteoporótica de fêmur no sistema suplementar de saúde brasileiro. Arq Bras Endocrinol Metab. 2005;49(6):897-901.

12. Pedroni MA. Avaliação da força de flexão em três pontos sobre o calo ósseo nas fraturas diafisárias de fêmures de ratos fixadas com dois diâmetros de hastes diferentes [dissertação]. Curitiba: Pontifícia Universidade Católica do Paraná; 2005.

13. Singh M, Nagrath AR, Maini PS. Changes in trabecular pattern of the upper end of the femur as an index of osteoporosis. J Bone Joint Surg Am. 1970;52( 3):457-67.

14. Nordin BE, Polley KJ. Metabolic consequences of the menopause. A cross-sectional, longitudinal, and intervention study on 557 normal postmenopausal women. Calcif Tissue Int. 1987;41(Suppl 1):S1-59.

15. Brasil: Tabua de vida. Internet. Disponivel: wuw.ibge.gov.br/home/estatistica/populacao/tabuadevida/2006/feminino.pdf Acesso: 22 out 2008.

16. Stenstrom M, Olander B, Lehto-Axtelius D, Madsen JE, Nordsletten L, Carlsson G. Bone mineral density and bone structure variables as predictors of bone strength: an analysis using computerized microtomography and gastrectomy-induced osteopenia in the rat. J Biomech. 2000;33(3):289-97.

17. Cardoso Netto C, Franco M, Cunha MSCA, Miyasaka CK. Efeitos da ovariectomia ricos que permitem comparações e estudos estatísticos com especificidade segura ${ }^{(12)}$.

Neste estudo, apesar de os fêmures dos animais do grupo controle apresentarem densidade tomográfica superior à dos outros dois grupos, não se observou correlação estatisticamente significante ao se comparar a força de ruptura com a força máxima, nos três grupos.

Estudos comprovam que a terapia de reposição hormonal produz alterações benéficas em animais submetidos a ela. Observa-se uma mudança na composição mineral do osso, principalmente em áreas críticas a fraturas, principalmente em ossos longos ${ }^{(29-31)}$.

O conhecimento de mecanismos de prevenção da fratura e mesmo de tratamento dela poderá contribuir para vida com melhor qualidade para a população de idosos.

\section{CONCLUSÕES}

O presente estudo permite concluir que a terapia de reposição hormonal, em ratas, leva a aumento significativo da resistência óssea. O exame tomográfico utilizado não demonstrou alterações significantes da densidade mineral óssea. experimental no metabolismo ósseo de ratas Wistar adultas: um modelo para estudo da osteoporose. Rev Cienc Med Biol. 2006;5(3):231-8.

18. Probst A, Jansen $H$, Ladas $A$, Spiegel $H U$. Callus formation and fixation rigidity: a fracture model in rats. J Orthop Res. 1999;17(2):256-60.

19. Oliveira LG. Osteoporose: guia para diagnóstico, prevenção e tratamento. Rio de Janeiro: Revinter; 2002.

20. Marshall D, Johnell 0 , Wedel $\mathrm{H}$. Meta-analysis of how well measures of bone mineral density predict occurrence of osteoporotic fractures. BMJ. 1996;312(7041):1254-9.

21. Horton JA, Murray GM, Spadaro JA, Margulies BS, Allen MJ, Damron TA. Precision and accuracy of DXA and pQCT for densitometry of the rat femur. J Clin Densitom. 2003;6(4):381-90.

22. Schmidt C, Priemel M, Kohler T, Weusten A, Müller R, Amling M, et al. Precision and accuracy of peripheral quantitative computed tomography (pQCT) in the mouse skeleton compared with histology and microcomputed tomography (microCT). J Bone Miner Res. 2003;18(8):1486-96.

23. Chen Q, Kaji H, lu MF, Nomura R, Sowa $H$, Yamauchi M, et al. Effects of an excess and a deficiency of endogenous parathyroid hormone on volumetric bone mineral density and bone geometry determined by peripheral quantitative computed tomography in female subjects. J Clin Endocrinol Metab._2003;88(10):4655-8.

24. Xiang A, Kanematsu M, Mitamura M, Kikkawa H, Asano S, Kinoshita M. Analysis of change patterns of microcomputed tomography 3-dimensional bone parameters as a high-throughput tool to evaluate antiosteoporotic effects of agents at an early stage of ovariectomy-induced osteoporosis in mice. Invest Radiol. 2006;41(9):704-12.

25. Ammann P. Determining factors of bone mechanical resistance. Therapie. 2003;58(5):403-7.

26. Carvalho MI. Osteoporose: a visão do ortopedista. Rev Bras Ortop. 2006;41(4):91-7.

27. Gabet Y, Müller R, Levy J, Dimarchi R, Chorev M, Bab I, et al. Parathyroid hormone 1-34 enhances titanium implant anchorage in low-density trabecular bone: a correlative micro-computed tomographic and biomechanical analysis. Bone. 2006;39(2):276-82.

28. Yuehuei H, Draughn RA. Mechanical testing of bone and the bone-implant interface. Boca Raton: CRC Press LLC; 2000. p. 207-17.

29. Ynsa MD, Ager FJ, Alves LC, Zubeldia MA, Millán JC, Pinheiro T. Elemental distributions in femoral bone of rat under osteoporosis preventive treatments. J Microsc. 2006;224(Pt 3):298-305.

30. van Geel TA, Geusens PP, Nagtzaam IF, van der Voort DJ, Schreurs CM, Rinkens $\mathrm{PE}$, et al. Risk factors for clinical fractures among postmenopausal women: a 10-year prospective study. Menopause Int. 2007;13(3):110-5.

31. Mosekilde L, Vestergaard P, Langdahl B. Fracture prevention in postmenopausal women. Am Fam Physician. 2008;77(10):1447-8. 